\title{
Salud y medio ambiente en el Perú actual
}

\author{
Health and environment in Peru: Current situation
}

Correspondencia Alberto Zolezzi F. zolezzi3z2011@gmail.com

Recibido: 05/06/2017 Aprobado: 07/06/2017

Citar como: Zolezzi A. Salud y medio ambiente en el Perú actual [editorial]. Acta Med Peru. 2017;34(2):79-81

\author{
Alberto Zolezzi F.1,a \\ 1 Hospital María Auxiliadora \\ a Director Acta Médica Peruana
}

La salud de las personas está basada en muchos factores. Aspectos que siempre se estudian son los antecedentes familiares y genéticos que pueda tener cada persona. Esto tiene tal importancia que el consejo genético determina aspectos éticos en lo concerniente al riesgo de tener hijos con enfermedades incapacitantes, incurables e inclusive mortales.

Otro aspecto en la salud de las personas es la alimentación, y es reconocido como diversos tipos de dieta pueden contribuir al desarrollo de problemas de salud v.g. consumo de sal e hipertensión arterial, grasas e hiperlipidemias y obesidad. Azucares y predisposición a la diabetes; y un sinnúmero de otras patologías relacionadas con nuestro tipo de alimentación. Los estados ante los hechos de esta influencia nociva de los comportamientos dietéticos en la población, dictan normas que tratan de orientar la alimentación. La reciente reglamentación de la Ley de Alimentación Saludable pone en actualidad la importante cuestión de los factores que pueden afectar a la salud. Por ello, una adecuada educación en nuestra alimentación es necesaria. Hay controversias sobre este tema e indudablemente la salud de cada uno de nosotros no se rige por leyes, sino por el conocimiento y toma de conciencia de lo que es alimentarse saludablemente.

Es importante en la salud el medio ambiente que nos rodea. Esto es muy amplio y genera reacciones desde el punto de vista local hasta llegar a compromisos globales como el dado por el convenio de protección del medio ambiente y los acuerdos de Paris del 2015.

Si bien el cambio climático, puede haber exacerbado factores medio ambientales que afectan nuestra salud, como son las lluvias e inundaciones y cambios extremos de temperatura; nuestro medio ambiente sin estos cambios dramáticos también ejerce acción sobre nuestro estado de salud.

En esta editorial queremos recordar en forma general como el medio ambiente puede afectar la salud y esto es una voz de alerta a la situación de nuestro país en la protección del medio ambiente.

Factores medioambientales que afectan la salud ${ }^{[1]}$ :

- La relación entre la contaminación del aire exterior,

- En ambientes interiores el humo del tabaco (HT) es el más frecuente factor contaminante,

- La disminución de la capa de ozono estratosférico,

- La exposición a sustancias y preparados químicos peligrosos,

- La exposición al ruido,

- El agua de consumo no potable o contaminada,

- Otros problemas emergentes son la radiactividad natural y artificial.

Se ha estimado que en los países un $20 \%$ de la incidencia total de enfermedades puede atribuirse a factores medioambientales. 


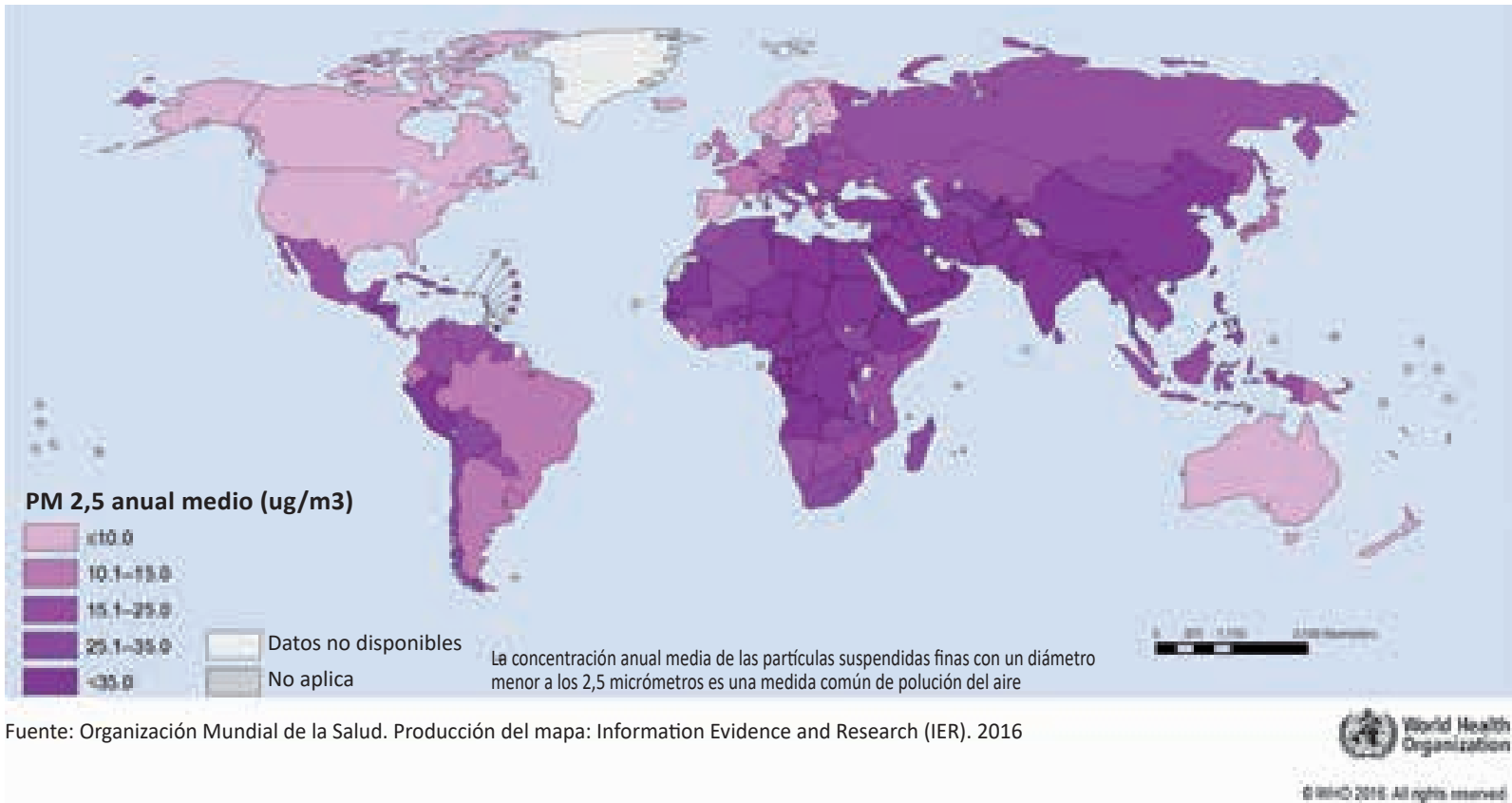

Figura 1. Concentración anual promedio de materia particulada fina (PM 2,5) en áreas urbanas $\left(\mu \mathrm{g} / \mathrm{m}^{3}\right), 2014$.

Las enfermedades respiratorias, el asma y las alergias están asociadas con la contaminación del aire externo e interno. Los agentes ambientales implicados son los óxidos de nitrógeno y azufre, las partículas en suspensión, ozono, metales, compuestos orgánicos volátiles (COV) y los hidrocarburos. En ambientes interiores el humo del tabaco (HT) es el más frecuente.

El HT aumenta el riesgo de cáncer en un 20-30 \% entre los no fumadores.

Las evidencias del impacto del cambio climático sobre la salud son cada día más consistentes ${ }^{[2,3]}$. En relación con ello recientes trabajos ha concluido que nuestro país es uno de los más vulnerables al cambio climático y se está viendo afectado por los impactos de este cambio. Los impactos sobre la salud humana se refieren a un aumento de la morbimortalidad por olas de calor que pueden ser más frecuentes en intensidad y duración los próximos años. Otros efectos son el aumento de la contaminación por partículas finas y ozono y la implantación de vectores subtropicales adaptados a sobrevivir en climas cálidos y más secos, lo que podrá aumentar la incidencia de enfermedades como el dengue, zika entre otros.

La disminución de la capa de ozono estratosférico y la exposición a radiaciones ultravioletas están asociadas a un aumento del cáncer de piel, cataratas y alteraciones del sistema inmunitario.

La exposición al ruido provoca trastornos auditivos, trastornos cardiovasculares, estrés, irritabilidad, alteraciones del sueño, gastos económicos (medidas de protección y aislamiento acústico], etc.

El agua de consumo puede transmitir numerosas enfermedades producidas por agentes microbiológicos y químicos. En nuestro país los brotes de enfermedades hídricas que se relacionan con abastecimientos de agua no apta para el consumo. El problema emergente en nuestro entorno son las enfermedades causadas por contaminantes químicos, ya sea por contaminación del agua en origen o bien debido a las características químicas del abastecimiento, por los materiales instalados en contacto con el agua de consumo, por las sustancias formadas como subproductos de reacción por la utilización de tratamientos químicos necesarios para la potabilización del agua, o por el mal mantenimiento o diseño de las instalaciones.

El denominador común de estas enfermedades es que en la mayoría de los casos el efecto sobre la salud no es inmediato, sino a mediano o largo plazo, dando como resultado enfermedades de tipo degenerativo en las que resulta muy difícil establecer relaciones de causalidad.

Uno de los aspectos más complicados es estimar que peso tienen los factores ambientales en la presencia de enfermedades a las que se atribuye influencia su desarrollo [4]. La Organización Mundial de la Salud (OMS) ha desarrollado estimados mundiales de porcentaje de enfermos con enfermedad del tracto respiratorio inferior agudo en niños (ETRI), enfermedad pulmonar obstructiva crónica (EPOC), cáncer de pulmón (CP), enfermedad cardiaca isquémica (ECI), y accidente cerebro vascular (ACV), según la población en riesgo y evaluando los niveles de partículas de $<2,5 \mu$ m en promedio en un año, midiendo niveles de fluctuación.

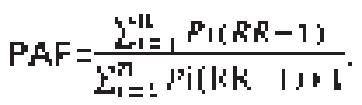

En esta fórmula algo compleja; el RR, se refiere al riesgo relativo de una enfermedad en relación a una exposición ambiental anual 
comparado a un valor control, i es el nivel de $\mathrm{PM}<2,5 \mu \mathrm{m}, \mathrm{Pi}$, es el porcentaje de población expuesta a esos valores de PM $<2,5 \mu \mathrm{m}$. Esto ha permitido, con los datos que dispone la OMS, hacer la estimación de los riesgos de las enfermedades antes mencionadas ${ }^{[4]}$ que son:

Nivel Mundial; $\quad$ ETRI 17\% EPOC 8\% CP 25\% ECI 15\% ACV 16\%. America Latina; ETRI $12 \%$ EPOC $2 \%$ CP 15\% ECI 12\% ACV $11 \%$.

En el Perú el año 2012 la OMS ha estimado que 163 casos de ETRI han sido consecuencia de las características de polución de nuestro aire. 128 casos de EPOC, 651 casos de CP, 1949 pacientes con $\mathrm{ECl}$ y 1348 pacientes con ACV. Son 4239 casos y ajustado por edades $17 / 100000$ habitantes.

Puede sonar una cantidad pequeña si sumamos el total de la población; pero esto es por año; con posibilidades a que si no nos preocupamos de nuestro ambiente esto puede aumentar. Y no debemos considerar que esto es consecuencia del cambio climático exclusivamente. En las ciudades, la densidad del tránsito con los gases de combustión, el combustible de fábricas, talleres o de hogares que usan combustibles tóxicos e incluso leña son una advertencia.

En este plano mundial elaborado por la $\mathrm{OMS}^{[5]}$ (Figura 1), sobre la presencia de partículas de $\leq 2,5 \mu \mathrm{m}$ en el aire exterior que están sobre los niveles considerados tolerables de $\leq 10 \mu \mathrm{g} / \mathrm{m}^{3}$, el Perú tiene el poco grato honor de estar entre los con mayor polución. Esta evaluación sobre el Perú tiene como sesgo que casi todo lo que se mide del Perú es Lima.
En los aspectos de la alimentación, la educación y adecuada información nutricional de los alimentos es de vital importancia. En los factores medio ambientales hay un adecuado conocimiento que altera la calidad del medio ambiente y es deber del estado, evitar se altere este medio ambiente tan delicado. A nivel multinacional el esfuerzo general tiene que agotarse para que los efectos sobre el cambio climático no se agraven.

El médico, tiene que luchar contra la contaminación de los autos que congestionan el tránsito de las grandes ciudades. Los humos de las fábricas; la contaminación del agua de los ríos, la contaminación sonora. El médico de hoy ya no es solo del hospital y el consultorio; tiene que buscar que el entorno sea también saludable.

\section{REFERENCIAS BIBLIOGRÁFICAS}

1. Vargas Marcos, F. La contaminación ambiental como factor determinante de la salud. Rev Esp Salud Publica. 2005;79(2):117-27.

2. Sánchez Zavaleta CA. Evolución del concepto de cambio climático y su impacto en la salud pública del Perú. Rev Peru Med Exp Salud Publica. 2016;33(1):128-38.

3. Figueroa R. Cambio climático y salud. Rev Peru Med Exp Salud Publica. 2016;33(1):7-9.

4. World Health Organization. Public Health Enviromental and Social determinants of health Program [Internet]. Geneva: WHO; c2017 [citado el 01 de junio de 2017]. Disponible en: http://gamapserver. who.int/mapLibrary/Files/Maps/Global_oap_pm25_2014.png

5. World Health Organization. Public Health Enviromental and Social determinants of health Program [Internet]. Geneva: WHO; c2017 [citado el 01 de junio de 2017]. Disponible en: http://gamapserver who.int/mapLibrary/Files/Maps/Global_oap_pm25_2014.png

\title{
Ahora puede enviar sus artículos para Acta Médica Peruana en nuestro Open Journal System:
}

\author{
www.amp.cmp.org.pe
}

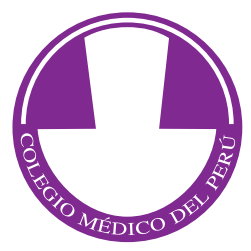

